\title{
What kind of processing is survival processing?
}

\section{Effects of different types of dual-task load on the survival processing effect}

\author{
Meike Kroneisen $^{1} \cdot$ Jan Rummel $^{2} \cdot$ Edgar Erdfelder $^{3}$
}

Published online: 1 August 2016

(C) Psychonomic Society, Inc. 2016

\begin{abstract}
Words judged for their relevance in a survival context are remembered better than words processed in non-survival contexts. This phenomenon is known as the survival processing effect. Recently, inconsistent results were reported on whether the size of the survival processing effect is affected by cognitive load. Whereas Kroneisen, Rummel, and Erdfelder (Memory 22: 92-102, 2014) observed that the survival processing effect vanishes under dual-task conditions, Stillman, Coane, Profaci, Howard, and Howard (Memory \& Cognition 42: 175-185, 2014, Experiment 1) found that the size of survival processing effect is essentially unaffected by a cognitively demanding secondary task. In three experiments, we investigated the differences between these studies to achieve a better understanding of dual-task effects on the survival-processing advantage. In the first experiment, we replicated Stillman et al.'s results using their dual-task conditions combined with a sample more than twice as large as theirs. In the second experiment, we compared dual-task conditions that differed regarding how strongly the secondary task taxed (a) working memory load (maintenance of one vs. several items) and (b) processing demands (switching vs. timesharing between tasks). A third experiment focussed on low (i.e., single-item) load under time-sharing processing conditions.
\end{abstract}

Meike Kroneisen

kroneisen@uni-landau.de

Edgar Erdfelder

erdfelder@uni-mannheim.de

1 Department of Psychology, University of Koblenz-Landau, Fortstraße 7, 76829 Landau, Germany

2 Department of Psychology, Heidelberg University, Hauptstrasse 47-51, 69117 Heidelberg, Germany

3 Department of Psychology, School of Social Sciences, University of Mannheim, Schloss, Ehrenhof-Ost, 68131 Mannheim, Germany
Results consistently showed that the survival processing effect persisted under low load but vanished when the number of items held in working memory increased beyond one, irrespective of processing demands. Implications of these findings for explanations of the survival-processing advantage are discussed.

Keywords Episodic memory $\cdot$ Evolution $\cdot$ Survival processing effect $\cdot$ Working memory load

Memory research has often focused on structural mechanisms, making use of more or less abstract materials in artificial learning environments. However, it seems unlikely that human memory evolved to learn, process, and store abstract information. If the evolution of human memory was shaped by the process of natural selection, then structural properties of memory should reflect their functionality (Tooby \& Cosmides, 1992). The selection pressure, that is, the adaptive problem to be solved, constrains how and why a structure develops and also the form it takes. Looking at our memory system, it seems implausible that its function is only to remember the past. It seems more plausible that we need to remember the past to predict the likelihood of events occurring in the future (Suddendorf \& Corballis, 1997; Tulving, 2002). Specifically, memory could be designed to retain information relevant for future survival, for example, by remembering the location of relevant food resources or potable water.

In line with this idea, Nairne, Thompson, and Pandeirada (2007) were able to demonstrate that verbal information processed in the context of an imagined ancestral survival scenario (i.e., being stranded in the grasslands of a foreign land) is recalled better than information encoded using alternative mnemonic procedures that proved to be highly effective. This phenomenon is known as the survival processing effect 
(SPE). In the typical experimental design, words are rated with respect to their relevance for an ancestral grassland survival scenario characterized by predators, lack of food, and lack of potable water. After a short distractor task, memory performance for the previously rated words is tested in a surprise free-recall test. Recall performance is typically much better in the survival group compared to various control groups, for example, groups that were required to rate the same words with respect to their pleasantness (e.g., Nairne et al., 2007; Nairne \& Pandeirada, \& Thompson, 2008) or their selfrelevance (Kostic, McFarlan, \& Cleary, 2012; Nairne et al., 2008; but see Klein, 2012), that got imagery instructions instead (Nairne \& Pandeirada, 2008), or that rated the relevance of the same words for a control scenario such as a moving scenario (e.g., Nairne et al., 2007) or emotionally more arousing control scenarios (e.g., Butler, Kang, \& Roediger, 2009). Moreover, one of the studies from Nairne et al. (2008) was also successfully replicated as part of the reproducibility project (Renkewitz \& Müller, 2015, July 13). Thus the SPE can be considered a very stable memory phenomenon that does not depend on comparisons with a specific control condition.

However, it is still an open debate which mechanisms drive the survival processing advantage (for reviews, see Erdfelder \& Kroneisen, 2014; Howe \& Otgaar, 2013; Kazanas \& Altarriba, 2015; Nairne \& Pandeirada, 2016). As outlined by Kroneisen et al. (2014) there are different proximate explanations of the effect. According to evolutionary explanations, the SPE reflects "hardwired" automatic processes based on evolved domain-specific cognitive modules. For example, Nairne, Vasconcelos, and Pandeirada (2011) argued that the SPE can be seen as evidence that human learning and memory systems have been selectively tuned during evolution to process and retain information that is relevant to fitness. One implication of the evolutionary account is that the survival processing advantage should be very general and thus hold across a broad range of cultural and situational context conditions. In line with this prediction, the SPE was indeed found in all countries in which it was investigated so far (USA: e.g., Nairne et al., 2007; Germany: e.g., Kroneisen \& Erdfelder, 2011; UK: e.g., Howe \& Derbish, 2010; The Netherlands: e.g., Otgaar, Smeets, \& van Bergen, 2010; Japan: e.g., Nouchi, 2012), and it also proved to be particularly robust across a wide range of contexts. Moreover, the SPE was replicated both in children (Aslan \& Bäuml 2012; Otgaar \& Smeets, 2010, Exp. 2) and in older adults (Nouchi, 2012; Yang, Lau, \& Truong, 2014; but see Otgaar, Jelicic \& Smeets, 2015, and Stillman, Coane, Profaci, Howard, \& Howard, 2014 , for different results). It was also found when replacing words with other to-be-remembered materials, for example, pictures (e.g., Otgaar, Smeets, \& van Bergen, 2010). Thus, there is little doubt that the SPE is a robust and rather general memory phenomenon as predicted by evolutionary accounts (cf. Erdfelder \& Kroneisen, 2014; Kroneisen \& Erdfelder, 2016; Nairne \& Pandeirada, 2016).

Evolutionary explanations stand in stark contrast to explanations arguing that survival processing may enhance memory because it recruits a powerful set of domaingeneral encoding processes more than typical control conditions do. One example is the richness of encoding (RE) hypothesis (Kroneisen \& Erdfelder, 2011; Röer, Bell, \& Buchner, 2013). According to this hypothesis, survival relevance ratings trigger richness of encoding, that is, each rated object is cognitively linked to a variety of possible functions it can have in ancestral survival contexts, both standard and atypical functions. By implication, thinking about possible survival functions of objects leads to highly distinctive and unique memory representations. In line with this hypothesis, Röer et al. (2013) found that SPE strength increases with the number of unique relevance arguments generated per item. Along the same lines, Bell, Röer, and Buchner (2015) further showed that rating the usefulness of objects compared to rating the dangerousness of objects in a survival scenario leads to better recall. Obviously, thinking about functions of objects (thus increasing the richness of encoding) results in better memory performance than thinking about the strength of emotions elicited by objects, as predicted by the RE hypothesis.

Domain-general "deep" encoding processes such as elaboration and distinctive processing assumed in the RE hypothesis involve consciously controlled forms of encoding that are cognitively demanding. By implication, they require selective attention and working memory capacity. Hence, the SPE should diminish or perhaps even vanish when working memory resources are scarce. This conflicts with the evolutionary view that implies stability of the survival processing advantage - as an evolved adaptation - irrespective of working memory resources. Evolutionary accounts often assume that psychological adaptations work automatically and unconsciously, that is, even if working memory resources are scarce. Fodor (1983), for example, claimed that psychological adaptations should meet the criteria automaticity, domain specificity, encapsulation, inaccessibility to consciousness, speed, shallow outputs, fixed brain location, and characteristic breakdown patterns. Admittedly, not all scholars agree with these criteria (see, e.g., Barrett \& Kurzban, 2006; Jung, Ruthruff, Tybur, Gaspelin, \& Miller, 2012). Nevertheless, if the survival processing advantage can be traced back to an evolved adaptation although it requires working memory resources, the least we would expect is that survival processing should be prioritized by default when several demanding tasks are processed simultaneously (see also Kroneisen et al., 2014, for a detailed discussion). Hence, the SPE should still persist under working memory load, in exchange for a deterioration in secondary task performance compared to control conditions. 
There are several ways to test these conflicting predictions against each other. One option is to assess the strength of the SPE in populations known to have lower working memory resources compared to healthy young adults, for example, older adults. According to evolutionary accounts, the SPE should be unaffected in older adults whereas it should be reduced according to the RE hypothesis. The available evidence is more in agreement with the latter prediction. As outlined above, Nouchi (2012) and Yang, Lau, and Truong (2014) found a significant SPE in older adults, too. However, in line with the RE hypothesis, in the experiment by Nouchi (2012) this effect was smaller in older than in younger adults as indicated by a significant interaction. Clear-cut results were also observed by Stillman et al. (2014): In none of their three experiments was there any evidence for a significant SPE in older adults. Similarly, there was also no SPE for older adults in a recent study by Otgaar, Jelicic, and Smeets (2015). However, despite these unequivocal results, one should be reluctant to discard the evolutionary account based on age group comparisons only, because young and older adults differ on many dimensions of which working memory capacity is only one. Without additional evidence, it remains unclear whether the often weaker (or even absent) SPE in older adults is, in fact, due to reduced working memory resources.

A more direct way to assess the causal role of working memory capacity in the SPE is to manipulate processing demands and working memory load experimentally using healthy young adults as participants only. Here, the current evidence is mixed. Kroneisen et al. (2014) found no survival processing advantage when the survival task was combined with an auditory continuous choice reaction time (CRT) task (cf. Naveh-Benjamin, Guez, \& Marom, 2003). Participants in the experiment by Kroneisen et al. (2014) rated words for their relevance to either a survival or a moving scenario under either standard conditions or dual-task conditions. Participants in the dual-task conditions were presented with a random sequence of high- and low-pitch tones via headphones while rating words with regard to their relevance for either the survival or the moving scenario. They were instructed to press the spacebar whenever the same tone was presented three times in a row.

In contrast to Kroneisen et al. (2014), Stillman et al. (2014, Exp. 1, young adults) were able to detect a significant survival processing advantage with the same two scenarios even when participants performed a secondary task during the relevance rating. In their Experiment 1, the size of the SPE was essentially unaffected by whether or not young adults processed the words in the survival or the moving scenario with or without a concurrent secondary task. Notably, however, their secondary task differed from the one used by Kroneisen et al. (2014). In Stillman and collaborators' secondary task, a high-pitched tone or a lowpitched tone was presented following each relevance rating. Participants were required to count the number of high-pitched tones and report them after all 32 words had been rated.
The purpose of the present paper is to clarify the reasons for the conflicting results of Kroneisen et al. (2014) and Stillman et al. (2014, Experiment 1), thereby contributing to the debate on whether survival processing is cognitively demanding as predicted by the RE hypothesis or automatic as predicted by evolutionary accounts of the SPE. The experiments of Kroneisen et al. (2014) and Stillman et al. (2014, Exp. 1, young adults) made use of the same basic $2 \times 2$ design, that is, type of scenario (survival vs. moving) was crossed with processing condition (single task vs. dual task). In this design, a significant interaction is indicative of a reduction in the size of the SPE under load. In addition, they also employed the same learning materials (i.e., word lists of Nairne et al., 2007). Apart from differences in language and nationality of the participants, the only important discrepancies between experiments concerned (1) the sample size across the four groups of the $2 \times 2$ design and (2) some core features of the secondary task used in the dual-task conditions of the design.

To isolate possible causes for the discrepant results, we first looked at differences in sample size. Whereas Kroneisen and collaborators analyzed data of 169 participants across the four groups, Stillman and collaborators' conclusions concerning young adults are based on 52 participants only. Thus, enhanced sampling error alone could be a reason for not finding a significant decrease of the SPE in the dual-task condition employed in Stillman et al.'s (2014) first experiment.

To test for the possibility that enhanced sampling error is responsible for the divergent results, an exact replication of Stillman et al.'s experiment seems necessary that has sufficient power to detect a reduction of the SPE under dual-task conditions if it exists. We thus opted to replicate their study with about 120 young adults across the four groups of the $2 \times 2$ between-subjects design, that is, approximately 30 participants per group. With this overall sample size and a type- 1 error risk of $\alpha=.05$, the power to detect a medium-sized interaction effect $(f$ $=.25$, see Cohen, 1988) of type of scenario with processing condition is close to the .80 power level recommended by Cohen (1988) (for exact results, see below). In contrast, Stillman and collaborators' experiment with the overall sample size of 52 had a power of only .42 for the same effect size.

There are two possible outcomes of the replication study. If the replication results in a significant reduction of SPE under the dual-task conditions employed by Stillman et al. (2014, Exp. 1), similar in size to the one previously observed by Kroneisen et al. (2014), then this would suggest that sampling error alone suffices as an explanation for Stillman and collaborators original results, making it obsolete to investigate differences between the secondary tasks of both experiments. If, in contrast, the replication is successful and again shows no significant reduction of the SPE under Stillman's dual-task conditions 
despite sufficient power, then this would suggest that differences in the dual-task conditions employed by Kroneisen et al. (2014) and Stillman et al. (2014, Exp. 1) are responsible for the discrepant results. Experiment 1 was designed to evaluate these possibilities.

\section{Experiment 1}

\section{Method}

\section{Participants}

Fifty-nine students from the University of Mannheim and 61 students from Heidelberg University participated for course credit or monetary compensation. Two participants were excluded due to low recall performance (number of recalled items below 5) or experience with the basic design of the survival-processing experiments. Thus, data analyses are based on the remaining 118 participants (27 male) who either received monetary incentives or course credit for compensation. Participants' age ranged from 18 to 36 years $(M=21.55$; $S D=3.33$ ). For a medium effect size $f=.25$ (Cohen, 1988), $\alpha$ $=.05$, and $N=118$, the power of the $2 \times 2$ interaction $F$-test is .77 (calculated with $\mathrm{G}^{*}$ Power, cf. Faul, Erdfelder, Buchner, \& Lang, 2009).

\section{Apparatus and materials}

Following Kroneisen et al. (2014), stimulus materials (i.e., words to be rated for their relevance) were obtained from Nairne et al.'s (2007) first experiment and translated into German. Thus, target words were 30 typical words from 30 unique categories. To absorb primacy and recency effects typically found in free recall, we added 12 buffer words drawn from the German version of the Battig and Montague norms (Mannhaupt, 1983), six at the beginning and six at the end of the list. All words, except the buffer words, were presented in random order. The survival and moving descriptions were German translations of those used by Nairne et al. (2007). The stimulus presentation was controlled by personal computers running Eprime 2.0 (Psychology Software Tools, Pittsburgh, PA, USA).

To replicate the results from Stillman et al. (2014), we developed a task analogous to the one they used. To this end, following the presentation and the rating of each individual word, participants heard either a high- or a low-pitched tone $(440 \mathrm{~Hz}$ and $330 \mathrm{~Hz}$ ) and were asked to count all high-pitched tones. Tones were presented immediately after the presentation of each individual word. Hence, 42 tones were presented in total. After the rating task, participants were asked to write down the overall number of high-pitched tones.

Design

A 2 (Type of scenario: survival vs. moving) $\times 2$ (Processing condition: single task vs. dual task) between-subjects design was used. Participants were randomly assigned to one of four groups (Group 1: survival scenario, single task, $n=31$, Group 2: moving scenario, single task, $n=29$, Group 3: survival scenario, dual task, $n=30$, Group 4: moving scenario, dual task, $n=28$ ).

The word-rating phase consisted of 42 trials, including the 12 buffer words at the beginning and at the end of the word list. Recall performance, response latencies, relevance ratings, and performance in the secondary task served as dependent variables.

\section{Procedure}

Depending upon the experimental condition, participants first read a scenario description (i.e., either the survival or the moving scenario depending on the experimental condition; see Kroneisen et al., 2014). They were then asked to rate the relevance of each item with respect to the relevant scenario. After two practice trials, half of the participants got the additional instruction to rate the items while performing a secondary task. Two different tones were presented to the participants via headphones. Their task was to count the overall number of the high-pitched tones. In the dual-task conditions, participants were asked to perform the secondary task without sacrificing the primary task (i.e., rating the relevance of the words presented). To familiarize participants with the tones, each of the two tones was presented once in the offset of the rating task. This was followed by a short practice phase where three words had to be rated for relevance under dual-task conditions. Subsequently, the main rating task (either with or without the secondary task, depending on experimental condition) started. Stimuli were presented individually for $5 \mathrm{~s}$ each. We asked participants to rate each word on a five-point scale, with 1 indicating "absolutely not relevant" and 5 indicating "extremely relevant" for the given scenario. Participants were required to respond within 5 s. If they failed to respond in time, a warning message appeared. The actual rating task was preceded by two short practice trials. After the rating task, participants performed a distractor task (i.e., filling-in an unrelated questionnaire) for $12 \mathrm{~min}$ and were then unexpectedly prompted with a free recall test for the words previously processed in the relevance-rating task. For this recall test, a time limit of $8 \mathrm{~min}$ was set for all participants. The experiment took approximately $30-35 \mathrm{~min}$ in total. 


\section{Results}

The significance level was set to $\alpha=.05$ for all statistical tests.

\section{Recall performance}

Figure 1 displays the mean proportions of words correctly remembered in the free recall test and their standard errors for all groups. A 2 (type of scenario) $\times 2$ (processing condition) ANOVA for mean proportions of words recalled revealed a significant main effect of processing condition $(F(1$, $\left.114)=10.86, p=.001 ; \eta_{p}{ }^{2}=0.09\right)$. Under cognitive load, recall performance was generally worse (Fig. 1). In addition, there was a significant main effect of scenario $(F(1,114)=$ $\left.20.51, p<.001 ; \eta_{p}{ }^{2}=0.15\right)$, but no significant interaction $(F(1$, $\left.114)=0.30, p=.59 ; \eta_{p}{ }^{2}=0.003\right)$, indicating an equally strong survival processing advantage under both single and dual-task conditions ( $M_{\text {Survival; } \text { single }}=17.09, S D=3.11 ; M_{\text {Moving; } \text { single }}=$ $13.93, S D=3.60 ; M_{\text {Survival; dual }}=14.70, S D=3.55 ; M_{\text {Moving; }}$; dual $=12.21, S D=3.27$ )

\section{Response times}

We also analyzed the response times of the rating task to see whether they were affected by the processing conditions. Table 1 shows the median response times of the participants' ratings for all conditions. A 2 (type of scenario: survival vs. moving) $\times 2$ (processing condition: single vs. dual task) ANOVA of response times showed a significant main effect of scenario, $F(1,114)=5.17, p=.03 ; \eta_{p}{ }^{2}=0.04$, indicating that ratings took longer in the survival condition. There was no significant main effect of processing condition $(F(1,114)=$ $\left.1.42, p=.23 ; \eta_{p}{ }^{2}=0.01\right)$ nor a significant interaction $\left(F(1,114)=0.30, p=.59 ; \eta_{p}^{2}=0.003\right)$.

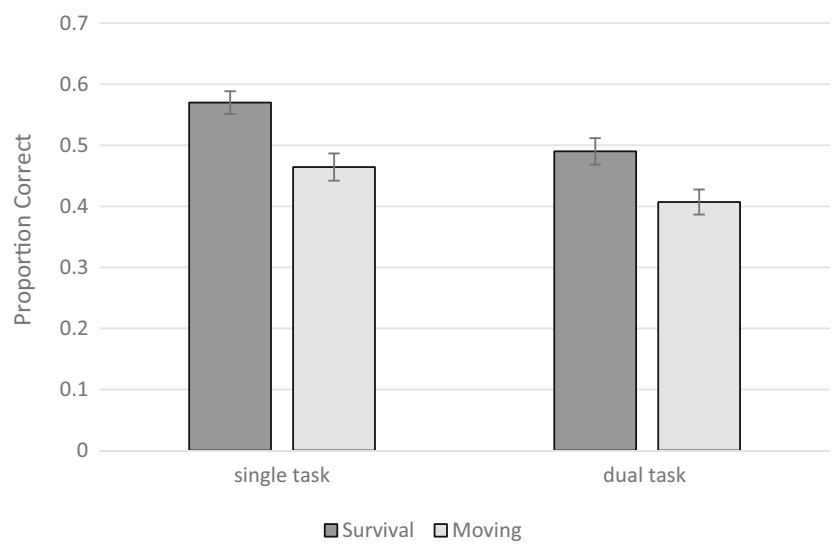

Fig. 1 Mean proportion of correct recall for each scenario, separately for each group in Experiment 1. The error bars represent standard errors of the means
Table 1 Means (M) and standard errors (SEMs) of participants' median response times, and participants' relevance ratings (relevance rating: $1=$ "absolutely not relevant", 5 = "extremely relevant") in Experiment 1

\begin{tabular}{|c|c|c|c|c|}
\hline \multirow[b]{2}{*}{ Condition } & \multicolumn{2}{|c|}{ Rating latencies (ms) } & \multicolumn{2}{|c|}{ Ratings } \\
\hline & M & SEM & M & SEM \\
\hline \multicolumn{5}{|l|}{ Single task } \\
\hline Survival & $2,206.24$ & 79.12 & 2.95 & 0.10 \\
\hline Moving & $2,052.71$ & 126.99 & 2.62 & 0.10 \\
\hline \multicolumn{5}{|l|}{ Dual task } \\
\hline Survival & $2,360.25$ & 69.67 & 2.87 & 0.12 \\
\hline Moving & $2,110.36$ & 66.09 & 2.61 & 0.10 \\
\hline
\end{tabular}

\section{Rating-task results}

To test whether participants' ratings differ between scenarios or processing conditions, we compared the mean rating scores between conditions. Relevance ratings were provided for $98.39 \%$ of the presented words. As Table 1 shows, average ratings were higher for the survival scenario in comparison to the moving scenario. An ANOVA revealed a significant main effect of scenario $(F(1,114)=$ 8.55, $\left.p=.004 ; \eta_{p}{ }^{2}=0.07\right)$, but neither a significant main effect of processing condition $(F(1,114)=0.22, p=.64$; $\left.\eta_{p}{ }^{2}=0.002\right)$ nor a significant interaction between scenario and processing conditions $\left(F(1,114)=0.13, p=.72 ; \eta_{p}{ }^{2}\right.$ $=0.001$ ).

As common in analyses of the SPE, the data were also analyzed to determine possible congruity effects, that is, whether items with higher ratings were also better remembered. Figure 2 shows the proportion of words recalled correctly in the free recall test as a function of initial rating, type of scenario, and cognitive load. For all conditions, a significant correlation between ratings and the proportion of words recalled was found. Controlling for overall recall performance of the participants, the partial correlation between rating and recall rates was significant for the survival, single-task group $(r=.16$; $p<.001)$, the moving, single-task group $(r=.25 ; p<.001)$, the survival, dual-task group $(r=.21 ; p<.001)$ and the moving, dual-task group $(r=.31 ; p<.001)$.

\section{Secondary-task performance}

To test whether involvement in the secondary task was comparable between both scenarios, we compared the number of correct responses to the secondary task in the survival and in the moving group. There was no performance difference between both conditions, $t(56)=0.81$, $p=.42$, two-tailed, $\eta_{p}{ }^{2}=0.01$. Thus, there was no 


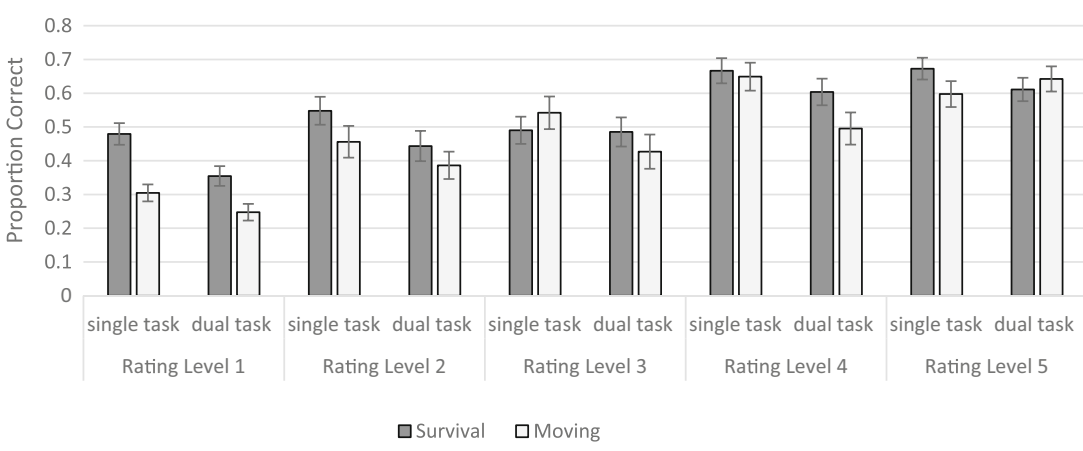

Fig. 2 Mean proportions of correct recall for each scenario, separately for each rating category in Experiment 1. The error bars represent standard errors of the means

evidence that participants in the survival group prioritized the survival-rating task at the cost of the secondary task.

\section{Discussion}

In this experiment, we replicated the effect found by Stillman et al. (2014) using a sample more than twice as large as the original sample. We combined the standard survival and moving scenarios with Stillman and collaborators' working memory load manipulation. Both scenarios have often been used to assess the size of the survival-processing effect (e.g., Nairne et al., 2007). In line with previous research, the secondary task led to a decrease in overall recall performance (e.g., Craik et al., 1996; Fernandes \& Moscovitch, 2000; Kroneisen et al., 2014; Stillman et al., 2014, Exp. 1). Replicating Stillman et al. (2014, Exp. 1), we observed SPEs of similar sizes in both the single-task and the dual-task conditions, as evident from a significant main effect of scenario and no interaction. Importantly, the nonsignificant interaction effect cannot be attributed to low statistical power.

The secondary task performance also did not differ between the survival and the moving conditions. Further, neither the relevance ratings nor their latencies varied as a function of single- and dual-task conditions. Thus, there was no evidence that people prioritized the primary task over the secondary task under any of the conditions.

However, we found a significant difference in response times between scenarios irrespective of processing conditions: Relevance ratings with respect to the moving scenario required less time than survival-relevance ratings. In addition, we also observed congruity effects between relevance ratings and memory performance, that is, higher perceived item relevance was associated with better recall later on. Similar effects were already obtained in previous studies (Butler et al., 2009; Kroneisen et al., 2013; Kroneisen et al., 2014; Kroneisen \& Erdfelder, 2011; but see Nairne \& Pandeirada, 2011).

In a nutshell, we replicated Stillman and collaborators' (2014, Exp. 1) results with a sample size more than twice as large as theirs, thus effectively ruling out the possibility that sampling error is responsible for the differences in results between Kroneisen et al. (2014) and Stillman et al. (2014, Exp. 1). This suggests that the discrepant results are due to differences in key features of the secondary tasks used in both experiments. However, what are the key features that might make a difference?

In the secondary task used by Stillman et al. (2014), a highpitched tone or a low-pitched tone was presented following each relevance rating. Participants were required to count the number of high-pitched tones and report them after all items had been rated. This secondary task thus allowed participants to switch between the primary task and the secondary task rather than to share processing-time between tasks, because the tones always occurred in the inter-trial-interval between the to-be-rated words. In addition, only a single number (i.e., the total score) had to be kept in active working memory and updated every time the next high-pitched tone was presented. By contrast, in the secondary task from Kroneisen et al. (2014) participants were presented with a random tone sequence consisting of two different auditory tones. Their task was to press the spacebar whenever the same tone was presented three times in a row. This CRT task was timed independently from the rating task so that participants could not simply switch back and forth between the primary task (the relevance rating) and the secondary task (the response to an auditory stimulus). Rather, participants were forced to perform both tasks concurrently all the time, that is, to share processing time between both tasks. In addition, the amount of information to be held in working memory was larger than in Stillman's study, as it involved the previous two tones that had to be updated every time a new tone was presented.

In Experiment 2 we further investigated the effects of two crucial differences between the secondary tasks of Kroneisen et al. (2014) and Stillman et al. (2014) on the size of the SPE, that is (1) the necessity of time-sharing versus switching between the primary and the secondary task and (2) the amount of information to be maintained and updated in working memory (two items vs. one item). 


\section{Experiment 2}

To assess how these different key features of the secondary tasks affect survival processing, we compared recall rates for the standard survival and moving conditions (Nairne et al., 2007) under three different dual-task conditions in Experiment 2. All three secondary tasks were different versions of an auditory choice reaction time task (i.e., 1-back and 2-back tasks, cf. D'Esposito $\&$ Postle, 2002). The three tasks differed in (1) whether they require maintaining, retrieving, and updating a single item versus two items in working memory (i.e., low vs. high working memory load induced by 1-back vs. 2-back tasks, respectively) and (2) whether they involve switching versus time-sharing between primary and secondary tasks. If high concurrent working memory load is crucial for the elimination of the SPE as observed by Kroneisen et al. (2014), then we would expect the SPE to vanish in the 2-back tasks conditions but not in the one-back task condition. If, however, time-sharing between the primary and the secondary task is crucial, then we would expect the SPE to vanish in the condition involving time-sharing but not in the conditions requiring switching between primary and secondary tasks.

\section{Method}

\section{Participants}

A total of 105 students from the University of Mannheim and 81 students from Heidelberg University participated for course credit or monetary compensation. Seven participants were excluded due to low recall performances (number of recalled items below 5) or experience with the basic design of the survival-processing experiments. Thus, data analyses are based on the remaining 179 participants (39 male) who either received monetary incentives or course credit for compensation. Participants' age ranged from 18 to 34 years $(M=$ $21.59 ; S D=3.37)$.

\section{Apparatus and materials}

The same stimulus materials as in Experiment 1 were used for the word-rating task. Also, the scenario descriptions were identical to Experiment 1.

Three different secondary tasks were used. To manipulate the two crucial dual-task features we used a 1-back and two different 2-back secondary tasks (low load vs. high load conditions, respectively). Both the 1-back task and one of the two 2-back tasks required switching between primary and secondary tasks whereas the other 2-back task involved time-sharing between tasks (switching vs. time-sharing conditions, respectively).

In the 1-back task, either a high- or a low-pitched tone $(440 \mathrm{~Hz}$ and $330 \mathrm{~Hz}$ ) was presented for $1 \mathrm{~s}$, immediately following the presentation and rating of each word. Following tone presentation, participants were asked whether the tone just presented was the same as the one presented before or not (1-back task). After the participant provided the answer, the next word was presented. Tones were presented immediately after the presentation of the words, therefore 42 tones were presented in total. The first dual-task probe occurred after the second tone. We refer to this condition as the Low-load-switching condition.

Similar to the Low-load-switching condition, for the first 2back task either a high- or a low-pitched tone $(440 \mathrm{~Hz}$ and 330 $\mathrm{Hz}$ ) was presented immediately after the presentation of each word. After tone presentation, participants were asked whether the tone just presented was the same as the one presented two tones before (2-back task). Following the participant's answer, the next word was presented. Tones were presented immediately after the presentation of the words. Hence, 42 tones were presented in total. The first dual-task probe occurred after the third tone. We refer to this condition as the High-load-switching condition.

The third and final secondary task was a continuous CRT tasks that involved a sequential presentation of auditory tones by the computer independently from the relevance ratings, one at a time, and a manual response on a computer keyboard. Participants were presented with a random sequence of two tones differing in pitch $(440 \mathrm{~Hz}$ and $330 \mathrm{~Hz})$. Each tone lasted for $1 \mathrm{~s}$ followed by an inter-stimulus interval of two seconds. The participants' task was to press the spacebar whenever the tone just presented was the same as the one presented two tones before (2-back task). The to-be-rated words appeared on the screen randomly in between, without any fixed rule that would allow participants to switch between tasks. We refer to this condition as the High-load-time-sharing condition.

\section{Design}

A 2 (Type of scenario: survival vs. moving) $\times 3$ (Dual-task condition: low-load-switching, high-load-switch, high-loadtime-sharing) between-subjects design was used. Participants were randomly assigned to one of the six groups (Group 1: survival scenario, low-load-switching, $n=33$, Group 2: moving scenario, low-load-switching, $n=34$, Group 3: survival scenario, high-load-switching, $n=28$, Group 4: moving scenario, high-load-switching, $n=24$, Group 5: survival scenario, high-load-time-sharing, $n=29$, Group 6: moving scenario, high-load-time sharing, $n=31$ ).

The word-rating phase consisted of 42 trials, including the 12 buffer words at the beginning and at the end of the word list. Recall performance, response latencies, relevance ratings, and performance in the secondary task served as dependent variables. 


\section{Procedure}

Similar to Experiment 1, depending upon the experimental condition, participants first read a scenario description (survival or moving depending on condition). They were then asked to rate the relevance of each item with respect to the relevant scenario. After two practice trails, the participants got the additional instruction to rate the items while performing a secondary task. Then, one of the three different secondary tasks (see above) was applied depending on the experimental condition. Secondary task responses were collected immediately after each tone. In all conditions, participants were instructed to perform the secondary task without sacrificing the primary task (i.e., rating the relevance of the words presented). To familiarize participants with the tones, each of the two tones was presented once in the offset of the rating task. This was followed by a short practice phase where three to four words (depending upon experimental conditions) had to be rated for relevance while performing the secondary task simultaneously. Subsequently, the main rating task (with a condition-dependent secondary task) started.

The rating task was identical to the one used in Experiment 1. After the rating task, participants performed the same 12-minutes distractor task as in Experiment 1 and were then unexpectedly prompted with a free recall test for the words previously processed in the relevance-rating task. For this recall test, a time limit of 8 min was set for all participants. The experiment took approximately $30-$ $35 \mathrm{~min}$ in total.

\section{Results}

Again, the significance level was set to $\alpha=.05$ for all statistical tests.

\section{Recall performance}

Figure 3 displays the mean proportions of correctly remembered words in the free recall test and their standard errors for all conditions $\left(M_{\text {Survival, low-load-switching }}=14.42, S D=3.67\right.$; $M_{\text {Moving,low-load-switching }}=11.29, S D=3.63 ; M_{\text {Survival, }}$ high-loadswitching $=11.89, S D=3.69 ; M_{\text {Moving,high-load-xwitching }}=11.29$, $S D=3.42 ; M_{\text {Survival, }}$ high-load-time-sharing $=10.72, S D=3.03$; $M_{\text {Moving, high-load-time-sharing }}=9.97, S D=3.66$ ). Since our predictions correspond to directed linear contrasts among the three levels of the Dual-Task factor and their interactions with the Scenario factor, a General Linear Model (GLM) contrast analysis of the two-factorial design was conducted. Planned comparisons based on the overall error term revealed a main effect of scenario $\left(t(173)=3.29, p<.001\right.$, one-tailed, $\eta_{p}{ }^{2}=$ $0.06)$, a main effect of high load vs. low load $(t(173)=-3.62$, $p<.001$, one-tailed, $\left.\eta_{p}{ }^{2}=0.07\right)$ and a main effect of switching vs. time-sharing $\left(t(173)=-2.10, p=.02\right.$, one-tailed, $\eta_{p}{ }^{2}=$
0.6

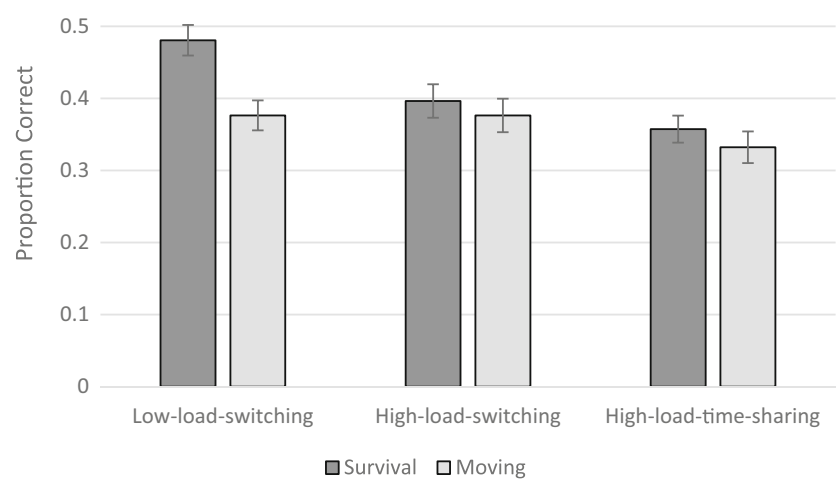

Fig. 3 Mean proportion of correct recall for each scenario, separately for each group in Experiment 2. The error bars represent standard errors of the means

0.02). Thus, on average, there was a survival processing advantage and also better performance under low-load and switching conditions compared to high-load and timesharing conditions, respectively. Importantly, however, a significant interaction occurred between scenario and the high vs. low load contrast $\left(t(173)=-1.89, p=.03\right.$, one-tailed, $\eta_{p}{ }^{2}=$ 0.02 ) revealing a significant reduction of the survival processing advantage under high-load compared to low-load conditions. In contrast, the interaction between scenario and the switching versus time-sharing contrast was not significant $\left(t(173)=0.69, p=.25\right.$, one-tailed, $\left.\eta_{p}{ }^{2}=0.003\right)$ indicating that the processing demands (i.e., task-switching vs. time-sharing) did not affect the size of the SPE significantly.

Subsequent simple-main effects analyses based on the overall error term revealed that the survival processing advantage in free recall was significant in the low-load-switching condition $\left(t(173)=3.62, p<.001\right.$, one-tailed, $\left.\eta_{p}{ }^{2}=0.07\right)$ but failed to be significant in both high-load conditions (highload-switching condition: $t(173)=0.61, p=.27$, one-tailed, $\eta_{p}{ }^{2}=0.002$; high-load-time-sharing condition: $t(173)=0.83$, $p=.20$, one-tailed, $\eta_{p}{ }^{2}=0.003$ ).

\section{Response times}

Again, we also checked whether there were differences in rating response times between the different dual-task conditions. Table 2 shows the median response times of the participants' ratings for all conditions. Planned comparisons based on the overall error term also revealed an overall effect of scenario $(t(173)=4.55, p<.001$, onetailed, $\eta_{p}{ }^{2}=0.11$ ) and of switching versus time-sharing $\left(t(173)=2.42, p=.01\right.$, one-tailed, $\left.\eta_{p}{ }^{2}=0.03\right)$, but not of high load versus low load $(t(173)=0.48, p=.32$, onetailed, $\left.\eta_{p}{ }^{2}=0.001\right)$. Thus, on average, participants spend more time with survival-relevance ratings than with moving-relevance ratings and with ratings in the Highload-time-sharing condition compared to the High-load- 
Table 2 Means (M) and standard errors (SEMs) of participants' median response times, and participants' relevance ratings (relevance rating: $1=$ "absolutely not relevant", 5 = "extremely relevant") in Experiment 2

\begin{tabular}{|c|c|c|c|c|}
\hline \multirow[b]{2}{*}{ Condition } & \multicolumn{2}{|c|}{ Rating latencies (ms) } & \multicolumn{2}{|c|}{ Ratings } \\
\hline & M & SEM & M & SEM \\
\hline \multicolumn{5}{|l|}{ Low-load-switching } \\
\hline Survival & $2,576.68$ & 92.86 & 2.98 & 0.09 \\
\hline Moving & $2,172.49$ & 73.72 & 2.72 & 0.07 \\
\hline \multicolumn{5}{|l|}{ High-load-switching } \\
\hline Survival & $2,530.43$ & 99.30 & 2.75 & 0.12 \\
\hline Moving & $2,302.42$ & 71.85 & 2.65 & 0.10 \\
\hline \multicolumn{5}{|c|}{ High-load-time-sharing } \\
\hline Survival & $2,798.69$ & 88.00 & 2.68 & 0.11 \\
\hline Moving & $2,464.23$ & 80.71 & 2.31 & 0.09 \\
\hline
\end{tabular}

switching condition. No significant interaction occurred between scenario and the high-versus-low-load contrast $\left(t(173)=-1.02, p=.16\right.$, one-tailed, $\left.\eta_{p}{ }^{2}=0.006\right)$. Also, the interaction between scenario and the switching-versustime-sharing contrast was not significant $(t(173)=0.60$, $p=.28$, one-tailed, $\eta_{p}{ }^{2}=0.002$ ).

\section{Rating-task results}

To test whether ratings differ between scenario types and dual-task conditions, we compared the mean rating scores across all conditions. Relevance ratings were provided for $97.49 \%$ of the presented words. We compared the mean rating scores across conditions. As Table 2 shows, average ratings were again higher for the survival scenario in comparison to the moving scenario. Planned comparisons based on the overall error term also revealed an overall effect of scenario $\left(t(173)=3.44, p<.001\right.$, one-tailed, $\eta_{p}{ }^{2}$
$=0.06)$ and of switching versus time-sharing $(t(173)=$ $2.05, p=.02$, one-tailed, $\eta_{p}{ }^{2}=0.02$ ), but not of high load versus low load $\left(t(173)=-1.57, p=.06\right.$, one-tailed, $\eta_{p}{ }^{2}=$ 0.01 ). Thus, on average, participants rated the words in the survival scenario as more relevant than the same words in the moving scenario. In addition, words were rated as more relevant in the High-load-time-sharing condition compared to the High-load-switching condition. No significant interaction occurred between scenario and the switching-vs-time-sharing contrast $(t(173)=1.38, p=.09$, one-tailed, $\eta_{p}{ }^{2}=0.01$ ). Also, the interaction between scenario and the high-versus-low-load contrast was not significant $\left(t(173)=-0.81, p=.21\right.$, one-tailed, $\left.\eta_{p}{ }^{2}=0.004\right)$.

The data were also analyzed to determine whether items with higher ratings were better remembered. Figure 4 shows the proportion of words recalled correctly in the free recall test as a function of initial rating, type of scenario, and cognitive load. Controlling for overall recall performance of the participants, the partial correlation between rating and recall rates was significant for all three survival-scenario groups (Lowload-switching condition: $r=.15 ; p<.001$; High-loadswitching condition: $r=.16 ; p<.001$; High-load-timesharing condition: $r=.20 ; p<.001)$ and also for all three moving-scenario groups (Low-load-switching condition: $r=$ $.20 ; p<.001$; High-load-switching condition: $r=.31 ; p<$ .001 ; High-load-time-sharing condition: $r=.28 ; p<.001$ ).

\section{Secondary-task performance}

Similar to Experiment 1, the performance in the secondary tasks was analyzed to test whether involvement in the secondary task was comparable between the scenarios. To this end, we compared the secondary task performance between the survival and the moving groups in all dual-task conditions. The number of correct responses in the secondary task was used to measure performance in the two switching conditions.

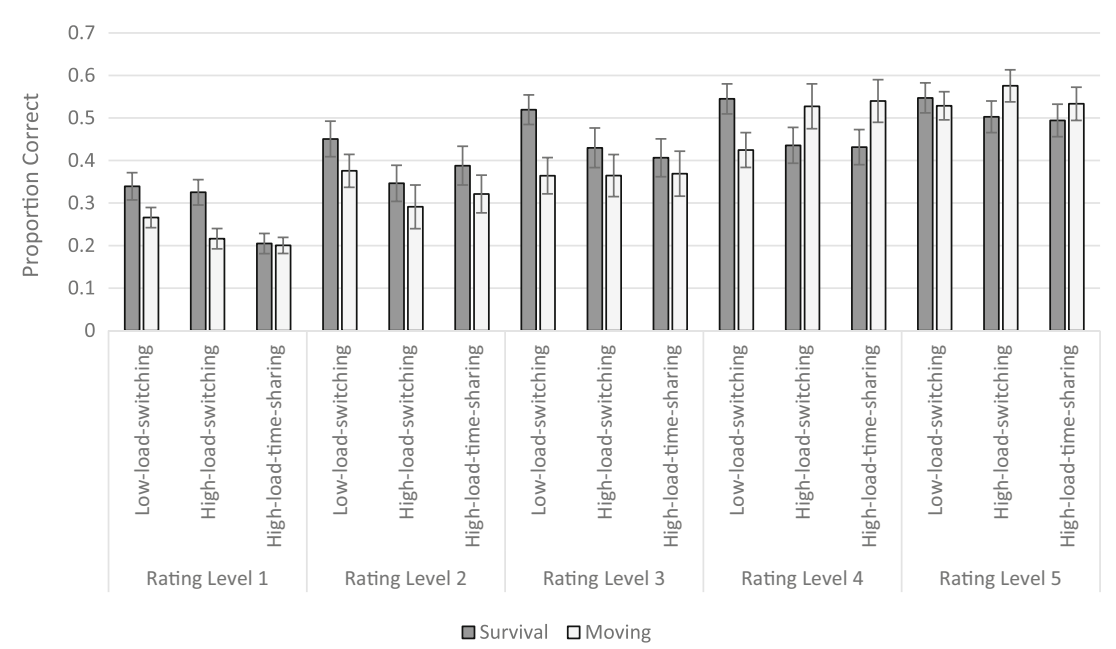

Fig. 4 Mean proportions of correct recall for each scenario, separately for each rating category in Experiment 2. The error bars represent standard errors of the means 
There was no difference in secondary task performance between scenarios, neither for the Low-load-switching condition $\left(t(65)=-1.88, p=.07\right.$, two-tailed, $\left.\eta_{p}{ }^{2}=0.05\right)$ nor for the Highload-switching condition $(t(50)=-0.73, p=.47$, two-tailed, $\left.\eta_{p}{ }^{2}=0.01\right)$. To measure secondary task performance in the High-load-time-sharing condition, we followed Kroneisen et al. (2014) and used the sensitivity measure of the twohigh threshold model (i.e., $p_{\mathrm{r}}=p$ [hit rate $]-p$ [false alarm rate]; Snodgrass \& Corwin, 1988). Again, no noteworthy difference between the survival and the moving condition was found in $p_{\mathrm{r}}\left(t(58)=-0.33, p=.75\right.$, two-tailed, $\left.\eta_{p}{ }^{2}=0.002\right)$. To conclude, across all dual-task conditions there was no evidence that participants in the survival group prioritized the survivalrating task at the cost of the secondary task.

\section{Discussion}

In Experiment 2, we analyzed the effect of different types of dual-task conditions on survival processing in more detail. To this end, we combined the standard survival and moving scenarios with several dual-task manipulations. In line with previous research and the results observed in Experiment 1, the secondary tasks led to a decrease in overall recall performance (e.g., Craik et al., 1996; Fernandes \& Moscovitch, 2000), showing that the manipulations were successful. However, when contrasting the low-load condition with the two high-load conditions, a significant main effect and a significant interaction between scenario and the high-versus-low-load contrast was detected. These findings indicate that maintaining und updating more than one item in working memory not only leads to an overall decline in memory but also diminishes the survival processing advantage compared to low-load conditions. In fact, simple main effects analyses revealed a significant SPE for the low-load-switching condition but no significant SPE for either of the two high-load conditions. Thus, as previously reported by Kroneisen et al. (2014), processing two items in working memory concurrently to the relevance rating task effectively eliminated the survival processing advantage. Importantly, it is unlikely that this null effect can be attributed to low statistical power. For a medium effect size $f^{2}=.15$ (Cohen, 1988), $\alpha=.05$, and $d f=173$, the power of our simple main effect $t$ tests still exceeds .99 (Faul et al., 2009). Thus, if there was a noticeable survival processing advantage under high working memory load, our test would have detected it with a high probability.

Moreover, for all secondary tasks the performance did not differ between the survival and the moving conditions. It was not the case that participants paid more attention to the rating task when reading a moving scenario before. Thus, there was again no evidence that the participants prioritized the primary task over the secondary task under survival conditions.

In line with several other studies, we found a significant difference in response times between scenarios. Across all conditions, participants spent more time for survivalrelevance ratings than for moving-relevance ratings. However, because these differences in processing times were observed under all three dual-task conditions to similar degrees, they cannot explain the effect of high versus low working memory load on the SPE.

In addition, we observed congruity effects between relevance ratings and memory performance. As in Experiment 1 , higher perceived item relevance was associated with better recall later on (see also Butler et al., 2009; Kroneisen et al., 2013; Kroneisen et al., 2014; Kroneisen \& Erdfelder, 2011; but see Nairne \& Pandeirada, 2011).

A drawback of Experiment 2 is that processing demands (time-sharing vs. task-switching) were manipulated (and found to be irrelevant) for the high-load condition but not for the low-load condition. Since we studied the low-load condition in combination with task-switching demands only, it is conceivable in principle that low load combined with time-sharing between tasks also eliminates the survival processing advantage, just as both high-load conditions did. Hence, given the evidence presented so far, we cannot rule out that time-sharing versus task-switching between tasks matters with respect to the SPE when working memory load is low. We conducted a third experiment to clarify this issue.

To assess how processing demands of secondary tasks affect survival processing under low-load conditions, we again compared recall rates for the standard survival and moving conditions (Nairne et al., 2007) in Experiment 3. In this experiment, the secondary task involved time-sharing between the primary and the secondary task combined with low working memory load induced by a 1-back task.

If only high concurrent working memory load is crucial for the elimination of the SPE as observed by Kroneisen et al. (2014), then the typical survival processing advantage should persist in Experiment 3. If, however, either high working memory load or time-sharing between tasks is sufficient for eliminating the survival processing advantage, then the SPE should virtually disappear in Experiment 3, too.

\section{Experiment 3}

\section{Method}

\section{Participants}

Fifty-nine students from the University of Mannheim participated for course credit or monetary compensation. Two participants were excluded because they did not meet the 
previously defined lower-bound for recall performance (i.e., at least five items recalled). Thus, data analyses are based on the remaining 57 participants ( 19 male) who either received monetary incentives or course credit for compensation. Participants' age ranged from $18-41$ years $(M=23.18 ; S D=4.92)$.

Prior to calculating the relevant $t$-test, we checked whether 57 participants are sufficient for detecting a SPE of the same size as observed in the low-load-time-switching condition of Experiment 2 with sufficient statistical power. Assuming Cohen's $d=.82$ (as suggested by the respective sample statistics of the previous Experiment 2) and given $\alpha=.05$, the power of a one-tailed two-groups $t$-test is .92 (Faul et al., 2009). Given that .80 is the generally recommended lower bound to statistical power (e.g., Cohen, 1988), we consider the power as adequate.

\section{Apparatus and materials}

The same stimulus materials as in Experiments 1 and 2 were used for the word-rating task. The scenario descriptions were also identical to Experiments 1 and 2.

We used a 1-back (low-load) time-sharing secondary task. This secondary task was a continuous CRT task that involved sequential presentation of auditory tones by the computer, independently from the timing of the relevance ratings and one at a time. Specifically, participants were presented with a random sequence of two tones differing in pitch $(440 \mathrm{~Hz}$ and $330 \mathrm{~Hz}$ ). Each tone lasted for $1 \mathrm{~s}$ followed by an inter-stimulus interval of $2 \mathrm{~s}$. The participants' task was to press the spacebar whenever the tone just presented was the same as the one presented before (1-back task). The to-be-rated words appeared on the screen randomly in between, without any fixed rule that would allow participants to switch between tasks. We refer to this condition as the Low-load-time-sharing condition.

\section{Design}

Participants were randomly assigned to one of the two groups (Group 1: survival scenario, low-load-sharing, $n=30$, Group 2: moving scenario, low-load-sharing, $n=27$ ).

The word-rating phase consisted of 42 trials, including six buffer words at the beginning and six buffer words at the end of the word list. Recall performance, response latencies, relevance ratings, and performance in the secondary task served as dependent variables.

\section{Procedure}

As in Experiments 1 and 2, participants first read a scenario description (survival or moving, depending on condition). They were then asked to rate the relevance of each item with respect to the respective scenario. After two practice trials, the participants got the additional instruction to rate the items while performing a secondary task. Secondary task responses were collected immediately after each tone. Again, participants were instructed to perform the secondary task without sacrificing the primary task (i.e., rating the relevance of the words presented). To familiarize participants with the tones, each of the two tones was presented once in the offset of the rating task. This was followed by a short practice phase where four words had to be rated for relevance while performing the secondary task simultaneously. Subsequently, the main rating task started.

The rating task was identical to the one used in Experiments 1 and 2. After the rating task, participants performed the same 12-min distractor task as in Experiments 1 and 2, followed by a surprise free recall test for the words previously processed in the relevance-rating task. For this recall test, a time limit of 8 min was set for all participants. The experiment took approximately $30-35 \mathrm{~min}$ in total.

\section{Results}

\section{Recall performance}

Figure 5 displays the mean proportions of words correctly remembered in the free recall test and their standard errors for both conditions. A $t$-test between the survival and the moving groups revealed a significant difference $(t(55)=$ 2.88, $p=.003$, one-tailed, $\left.\eta_{p}{ }^{2}=0.13\right)$. Participants in the survival group $\left(M_{\text {Survival; }}\right.$ low-load-sharing $=12.03, S D=$ 3.84) remembered more words than in the moving group ( $\left.M_{\text {Moving; } \text { low-load-sharing }}=9.56, S D=3.58\right)$, indicating a survival processing advantage.

\section{Response times}

We also analyzed the response times of the rating task to see whether they were affected by the processing conditions. Table 3 shows the median response times of the participants' ratings for all conditions. A $t$-test between the survival and the

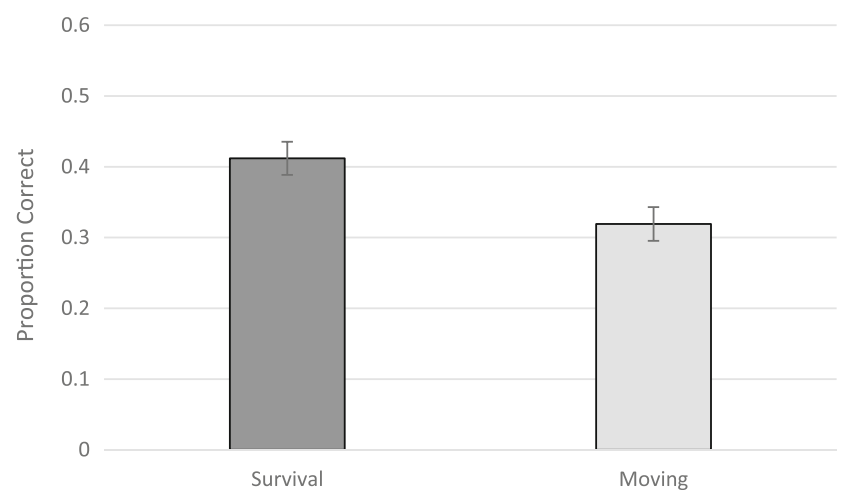

Fig. 5 Mean proportion of correct recall for each scenario, separately for both groups in Experiment 3. The error bars represent standard errors of the means 
Table 3 Means (M) and standard errors (SEMs) of participants' median response times, and participants' relevance ratings (relevance rating: $1=$ "absolutely not relevant", 5 = "extremely relevant") in Experiment 3

\begin{tabular}{llllll}
\hline & \multicolumn{2}{l}{ Rating latencies (ms) } & & \multicolumn{2}{l}{ Ratings } \\
\cline { 2 - 3 } \cline { 5 - 6 } Condition & $\mathrm{M}$ & & & $\mathrm{M}$ & SEM \\
\hline Low-load-sharing & & & & & \\
$\quad$ Survival & $2,674.62$ & 84.53 & & 3.05 & 0.09 \\
Moving & $2,381.74$ & 61.2 & & 2.48 & 0.08 \\
\hline
\end{tabular}

moving groups showed a significant effect of scenario, $t(55)=$ $2.75, p=.004$, one-tailed, $\eta_{p}{ }^{2}=0.12$, indicating longer rating latencies for the survival scenario condition.

\section{Rating-task results}

Again, we also tested whether participants' ratings differed between the scenarios. Relevance ratings were provided for 98.59 $\%$ of the presented words. As Table 3 shows, average ratings were higher for the survival scenario in comparison to the moving scenario. A $t$-test revealed a significant difference between both scenarios $\left(t(55)=4.50, p<.001\right.$, one-tailed, $\left.\eta_{p}{ }^{2}=0.27\right)$.

We also checked for possible congruity effects in Experiment 3, that is, whether items with higher ratings were also better remembered. Figure 6 shows the proportion of words recalled correctly in the free recall test as a function of initial ratings and type of scenario. Controlling for overall recall performance of the participants, the partial correlation between rating and recall rates was significant for both lowload-time-sharing conditions (survival group: $r=.11 ; p=$ .001 ; moving group: $r=.25 ; p<.001$ )

\section{Secondary-task performance}

To test whether involvement in the secondary task was comparable between both scenarios, we used the sensitivity measure of the two-high threshold model (i.e. $p_{\mathrm{r}}=p$ [hit rate] $-p$ [false alarm rate]; Snodgrass \& Corwin, 1988). Again, no difference between the survival and the moving condition was found in $p_{\mathrm{r}}\left(t(55)=0.16, p=.87\right.$, two-tailed, $\left.\eta_{p}{ }^{2}=0.0005\right)$. Thus, there was no evidence that participants in the survival group prioritized the survival-rating task at the cost of the secondary task.

\section{Discussion}

In the secondary task of Experiment 3, participants had to press the spacebar whenever a tone just presented was the same as the one presented before (1-back task). Concurrently, words had to be rated for their relevance with respect to the scenario. These words appeared on the screen randomly in between, without any fixed rule that would allow participants to switch between these tasks. Thus, participants were forced to share processing time between both types of tasks permanently. Importantly, we observed the typical survival processing advantage under these low-load-time-sharing conditions. Combined with the results of Experiment 2, this suggests that only working memory load is crucial for the emergence of the SPE: The survival processing benefit persists when only a single item is processed concurrently, whereas it vanishes when more than one item has to be maintained and updated in working memory. In contrast, processing demands such as time-sharing versus task-switching between primary and secondary tasks appear to be largely irrelevant with respect to the size of the SPE, at least when not more than two items are processed concurrently.

Replicating Experiments 1 and 2, secondary task performance did not differ between the survival and the moving conditions. Thus, there was no evidence that people prioritized the primary task over the secondary task under any of the conditions.

We found a significant difference in response times between scenarios: Relevance ratings with respect to the moving scenario required less time than survival-relevance ratings. In

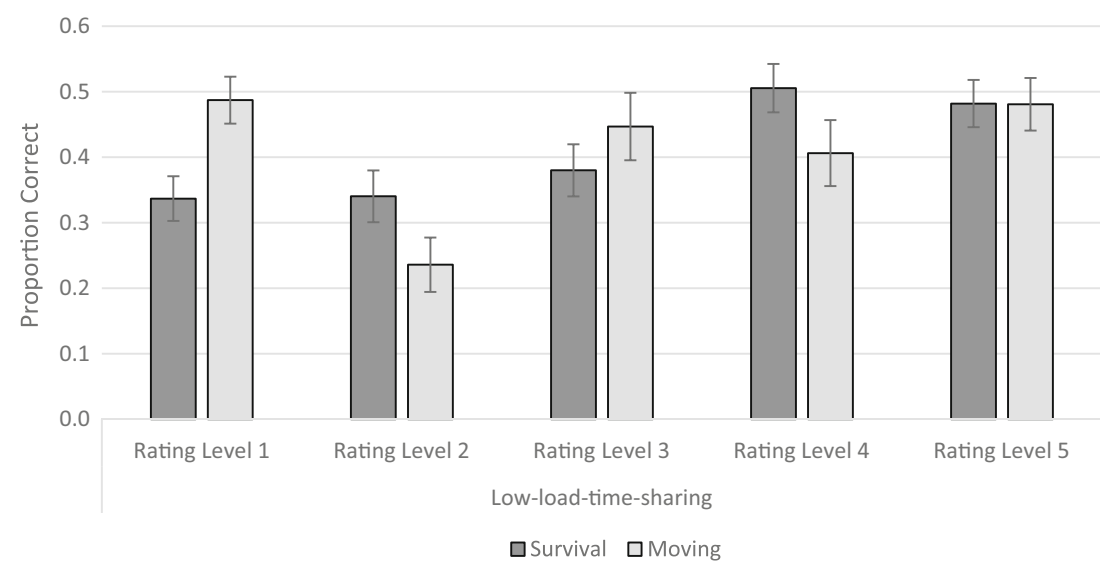

Fig. 6 Mean proportions of correct recall for each scenario, separately for each rating category in Experiment 3. The error bars represent standard errors of the means 
addition, we also observed congruity effects between relevance ratings and memory performance, that is, higher perceived item relevance was associated with better recall later on. Similar effects were already obtained in Experiments 1 and 2, as well as in other studies (e.g., Kroneisen \& Erdfelder, 2011). Although not strictly predicted, longer rating latencies in the survival scenario are in line with the assumption that domaingeneral encoding processes like richness of encoding are relevant mechanisms underlying the SPE. In general, distinctive processing takes time. Elaborative encoding often, but not always, requires more time than more shallow forms of encoding (for examples and exceptions, see Craik \& Tulving, 1975). Hence, from the richness-of-encoding perspective, it is not surprising to find longer rating latencies for those scenarios that are hypothesized to invite more elaborate forms of encoding. In addition, items that are congruent with or relevant for the processing task are remembered better. Assuming that higher relevance ratings correspond to more elaboration, these congruity effects are also perfectly in line with the assumption that domain-general encoding processes are important mechanisms to generate a SPE.

Notably, Experiment 3 can be seen as an extension of the previous Experiment 2. One way to integrate our Experiments 2 and 3 is to see them as disjoint parts of a completely crossed full-factorial 2 (Scenario: survival vs. moving) $\times 2$ (Workingmemory load: 2 items vs. 1 item) $\times 2$ (Processing demands: switching vs. sharing) design, thus enabling a corresponding 3 -factorial ANOVA. However, the results of such a joint analysis should be interpreted with caution, as participants were not fully randomized across all eight conditions. If participants from Experiments 2 and 3 were sampled from (slightly) different populations, unexpected effects can arise in the $2 \times 2 \times$ 2 joint ANOVA. Despite this caveat, it might be of interest to see that, in the joint ANOVA of Experiments 2 and 3, the expected two-way interaction of scenario with working memory load (high vs. low) was significant $(F(1,228)=5.36, p=$ $.02, \eta_{p}^{2}=0.023$ ), whereas the corresponding two-way interaction of scenario with processing demands (switching vs. sharing) was not $\left(F(1,228)=.15, p=.70, \eta_{p}{ }^{2}=0.001\right)$. Hence, this re-analysis is in line with our conclusion that working memory load moderates the SPE and that the processing demands in the dual-task condition - task switching versus time sharing - do not.

\section{General discussion}

To clarify discrepant results reported in the recent literature (Kroneisen et al., 2014; Stillman et al., 2014), we examined the influence of working memory load and dual-task processing demands on the survival processing advantage in free recall. In Experiment 1, we replicated Stillman and collaborators' (2014, Exp. 1) results with a larger sample, showing that sampling error alone cannot explain differences in results between Kroneisen et al. (2014) and Stillman et al. (2014, Exp. 1). This suggests that the discrepant results are due to differences in critical features of the secondary tasks used in both experiments, either (1) the possibility of switching between the primary and the secondary task (Stillman et al. 2014) versus the necessity of continuous time-sharing between both tasks (Kroneisen et al., 2014) or (2) maintaining and updating a single item in working memory (Stillman et al., 2014) versus maintaining and updating at least two independent items in working memory (Kroneisen et al., 2014). Experiment 2 was designed to assess the effect of these two factors on the size of the SPE. The results suggested that the amount of information in working memory is crucial for the SPE. Specifically, whereas the SPE was unaffected by the demands from keeping a single item in working memory, it essentially vanished when two items had to be processed concurrently. However, although Experiment 2 convincingly showed that time-sharing versus task-switching is irrelevant with respect to the SPE when two items are processed in working memory, it did not speak to the question of whether time-sharing would hamper survival processing under low-load condition, because low load was studied under task-switching conditions only. To close this gap, we conducted a third experiment in which we compared survival and moving scenarios under low-load time-sharing conditions. Resembling the results for the lowload-task-switching condition of Experiment 2, we found a significant SPE, indicating that task-switching versus timesharing between primary and secondary tasks does not affect the size of the SPE.

Notably, Nouchi (2013) reported results in line with those of Kroneisen et al. (2014) and the current Experiment 2. In Nouchi's (2013) memory load condition, participants were instructed to remember six randomly sampled digits while working on the relevance-rating task. The six digits were presented for 2 seconds before participants were asked to rate a word under survival processing or control conditions. Following the rating of each word, participants were required to recall the 6 digits. Thus, resembling Stillman et al.'s (2014) first experiment and our current Experiment 1, Nouchi's task allowed switching between the primary and the secondary task in the retrieval phase. However, the amount of information to be maintained in working memory was much larger in Nouchi's study (six digits rather than one item per trial). Interestingly, in line with Kroneisen et al. (2014) and the high-load conditions of our Experiment 2, Nouchi (2013) also failed to find the SPE when survival processing was combined with his memory load task. Thus, Nouchi's (2013) study provides converging evidence that the SPE emerges under noload or low-load conditions (i.e., concurrent processing of no more than a single item in working memory) whereas the SPE diminishes or even vanishes under high-load conditions (i.e., processing of more than one item in working memory). 
In contrast, it appears to be of minor importance for the size of the SPE whether the processing demands enforce strict timesharing or allow for switching between tasks such that retrieval from working memory is only required after providing a relevance rating. As shown by Nouchi (2013) and conceptually replicated in our current Experiment 2, high working memory load suffices to eliminate the SPE even under taskswitching conditions that are somewhat easier than timesharing conditions.

Putting the pieces together, we may conclude that despite the fact that maintaining and updating a single item in working memory hinders simultaneous encoding of a word list to some degree (as evidenced by lower recall rates for both the survival and the moving condition in the dual-task condition of Experiment 1) it does not affect the processing benefits causing the SPE, in young adults at least. In contrast, maintaining and updating several items in working memory concurrently with the survival relevance-rating task hampers the benefits associated with survival processing, so that the SPE virtually vanishes. Timing of retrieval from working memory for the secondary task - after versus while providing a relevance rating - plays a minor role only. Although time-sharing between primary and secondary tasks hinders overall list recall and increases the time required for relevance ratings more than task-switching does (see Table 2 and Fig. 3), the size of the SPE appears to be unaffected by retrieval timing.

On a more general level, our results show that different types of dual-task requirements may affect the SPE differently. This converges with results in other areas of memory research. For example, it has been found that different types of divided attention tasks may affect false memories differently (see e.g., Otgaar et al. 2012; Seamon et al., 2003). Similar effects were reported and discussed in implicit memory research (e.g., Mulligan, 1997; Mulligan \& Hornstein 2000).

It is important to note that - in line with previous research - we found significant SPEs across various conditions. This endorses the fact that the SPE is in general a robust and reproducible effect. Furthermore, our results show that the SPE is largely immune against low working-memory load from secondary tasks (e.g., a 1back task). Critically, however, the SPE virtually vanishes when the secondary task requires maintaining and updating more than one item in working memory. These findings, along with similar findings by Kroneisen et al. (2014) and Nouchi (2013) that we reviewed above, are difficult to reconcile with evolutionary accounts of the SPE but consistent with the RE account. A plausible interpretation is that high concurrent working memory load limits the cognitive resources available for generating and storing arguments about possible object functions in the relevance-rating task. It thus has effects similar to requiring participants to state a single use of an object (Kroneisen \& Erdfelder, 2011, Exp. 3) or to imagine a single use of an object only (Kroneisen, Erdfelder, \& Buchner, 2013), thus effectively eliminating particularly rich forms of encoding under survival relevance rating conditions. In line with this interpretation, we observed smaller differences between average survival- and moving-scenario rating latencies under high-load conditions (high-load-switching: $228 \mathrm{~ms}$, high-load-time-sharing: $334 \mathrm{~ms}$ ) compared to the low-load condition (404 $\mathrm{ms})$. However, given that the corresponding interaction failed to reach conventional levels of statistical significance, future research should further test whether the descriptive pattern of results indeed reflects a difference in quality of processing as presumed by the RE hypothesis.

In sum, the present findings not only replicate previous results but also explain apparent inconsistencies between these results. Moreover, in line with other recent work (e.g., Bell, Röer \& Buchner, 2013, 2015; Nouchi, 2013; Otgaar, Jelicec, \& Smeets, 2015; Kazanas, Van Valkenburg, \& Altarriba, 2015; Röer et al., 2013), they again corroborate elaborativeencoding explanations of the survival processing effect such as the richness-of-encoding framework and challenge evolutionary accounts like the selective-tuning hypothesis.

\section{References}

Aslan, A., \& Bäuml, K.-H. (2012). Adaptive memory: Young children show enhanced $\mathrm{r}$ etention of fitness-related information. Cognition, 122, 118-122. doi:10.1016/j.cognition.2011.10.001

Barrett, H. C., \& Kurzban, R. (2006). Modularity in cognition: Framing the debate. Psychological Review, 113, 628-647. doi:10.1037/0033295X.113.3.628

Bell, R., Röer, J. P., \& Buchner, A. (2013). Adaptive memory: The survival-processing memory advantage is not due to negativity or mortality salience. Memory \& Cognition, 41, 490-502. doi:10.3758 /s13421-012-0290-5

Bell, R., Röer, J. P., \& Buchner, A. (2015). Adaptive memory: Thinking about function. Journal of Experimental Psychology: Learning, Memory, and Cognition, 41, 1038-1048. doi:10.1037/xlm0000066

Butler, A. C., Kang, S. H. K., \& Roediger, H. L. (2009). Congruity effects between materials and processing tasks in the survival processing paradigm. Journal of Experimental Psychology: Learning, Memory, and Cognition, 35, 1477-1486. doi:10.1037/a0017024

Cohen, J. (1988). Statistical power analysis for the behavioral sciences. Hillsdale, NJ: Erlbaum.

Craik, F. I. M., \& Tulving, E. (1975). Depth of processing and the retention of words in episodic memory. Journal of Experimental Psychology: General, 104, 268-294.

Craik, F. I. M., Govoni, R., Naveh-Benjamin, M., \& Anderson, N. D. (1996). The effects of divided attention on encoding and retrieval processes in human memory. Journal of Experimental Psychology: General, 125, 159-180. doi:10.1080/713755914

D'Esposito, M., \& Postle, B. R. (2002). The organization of working memory function in lateral prefrontal cortex: Evidence from event-related functional MRI. In D. T. Stuss \& R. T. Knight (Eds.), Principles of frontal lobe function (pp. 168-187). Oxford, UK: Oxford University Press. doi:10.1093/acprof:oso/9780195134971.003.0011

Erdfelder, E., \& Kroneisen, M. (2014). Proximate cognitive mechanisms underlying the survival processing effect. In B. L. Schwartz, M. 
Howe, M. Toglia, \& H. Otgaar (Eds.), What is adaptive about adaptive memory? (pp. 172-198). New York: Oxford University Press. doi:10.1093/acprof:oso/9780199928057.003.0010

Faul, F., Erdfelder, E., Lang, A. G., \& Buchner, A. (2009). Statistical power analysis using $\mathrm{G}^{*}$ Power 3.1: Tests for correlation and regression analyses. Behavior Research Methods, 41, 1149-1160. doi:10.3758/BRM.41.4.1149

Fernandes, M. A., \& Moscovitch, M. (2000). Divided attention and memory: Evidence of substantial interference effects both at retrieval and encoding. Journal of Experimental Psychology: General, 129, 155-176.

Fodor, J. (1983). The modularity of mind. Cambridge, MA: MIT Press.

Howe, M. L., \& Derbish, M. H. (2010). On the susceptibility of adaptive memory to false memory illusions. Cognition, 115, 252-267. doi:10.1016/j.cognition.2009.12.016

Howe, M. L., \& Otgaar, H. (2013). Proximate mechanisms and the development of adaptive memory. Current Directions in Psychological Science, 22, 16-22. doi:10.1177/0963721412469397

Jung, K., Ruthruff, E., Tybur, J., Gaspelin, N., \& Miller, G. (2012). Perception of facial attractiveness requires some attentional resources: Implications for the "automaticity" of psychological adaptations. Evolution and Human Behavior, 33, 241-250. doi:10.1016/j.evolhumbehav.2011.10.001

Kazanas, S. A., \& Altarriba, J. (2015). The survival advantage: Underlying mechanisms and extant limitations. Evolutionary Psychology, 13, 360-396.

Kazanas, S. A., Van Valkenburg, K. M., \& Altarriba, J. (2015). Survival processing and the Stroop task: Does the survival advantage depend on deeper processing during encoding? Evolutionary Psychology, 13, 1-8. doi:10.1177/1474704915613912

Klein, S. B. (2012). A role for self-referential processing in tasks requiring participants to imagine survival on the savannah. Journal of Experimental Psychology: Learning, Memory, and Cognition, 38, 1234-1242. doi:10.1037/a0027636

Kostic, B., McFarlan, C. C., \& Cleary, A. M. (2012). Extensions of the survival advantage in memory: Examining the role of ancestral context and implied social isolation. Journal of Experimental Psychology: Learning, Memory, and Cognition, 38, 1091-1098. doi:10.1037/a0026974

Kroneisen, M., \& Erdfelder, E. (2011). On the plasticity of the survival processing effect. Journal of Experimental Psychology: Learning, Memory, and Cognition, 37, 1552-1563. doi:10.1037/a0024493

Kroneisen, M., Erdfelder, E., \& Buchner, A. (2013). The proximate memory mechanism underlying the survival processing effect: Richness of encoding or interactive imagery? Memory, 21, 494-502. doi:10.1080/09658211.2012.741603

Kroneisen, M., \& Erdfelder, E. (2016). Survival processing effect. In R. F. Pohl (Ed.), $C$ (2nd ed.). Hove: Psychology Press.

Kroneisen, M., Rummel, J., \& Erdfelder, E. (2014). Working memory load eliminates the survival processing effect. Memory, 22, 92-102. doi:10.1080/09658211.2013.815217

Mannhaupt, H. R. (1983). Produktionsnormen für verbale Reaktionen zu 40 geläufigen Kategorien. Sprache und Kognition, 4, 264-278.

Mulligan, N. W. (1997). Attention and implicit memory. The effect of varying attentional load on conceptual priming. Memory and Cognition, 25, 11-17. doi:10.3758/BF03197281

Mulligan, N. W., \& Hornstein, S. L. (2000). Attention and perceptual priming in the perceptual identification task. Journal of Experimental Psychology: Learning, Memory, and Cognition, 26, 626-637. doi:10.1037//0278-7393.26.3.626

Nairne, J. S., \& Pandeirada, J. N. S. (2008). Adaptive memory: Is survival processing special? Journal of Memory and Language, 59, 377385. doi:10.1016/j.jml.2008.06.001

Nairne, J. S., \& Pandeirada, J. N. S. (2011). Congruity effects in the survival processing paradigm. Journal of Experimental
Psychology: Learning, Memory, and Cognition, 37, 539-549. doi:10.1037/a0021960

Nairne, J. S., \& Pandeirada, J. N. S. (2016). Adaptive memory: The evolutionary significance of survival processing. Psychological Perspectives on Psychological Science.

Nairne, J. S., Pandeirada, J. N. S., \& Thompson, S. (2008). Adaptive memory: the comparative value of survival processing. Psychological Science, 19, 176-180. doi:10.1111/j.1467-9280.2008.02064.x

Nairne, J. S., Thompson, S. R., \& Pandeirada, J. N. S. (2007). Adaptive memory: survival processing enhances retention. Journal of Experimental Psychology: Learning, Memory, and Cognition, 33 , 263-273. doi:10.1037/0278-7393.33.2.263

Nairne, J. S., Vasconcelos, M., \& Pandeirada, J. N. S. (2011). Adaptive memory and learning. In N. M. Seel (Ed.), Encyclopedia of the sciences of learning (pp. 118-121). New York, NY: Springer.

Naveh-Benjamin, M., Guez, J., \& Marom, M. (2003). The effects of divided attention at encoding on item and associative memory. Memory \& Cognition, 31, 1021-1035. doi:10.3758/BF03196123

Nouchi, R. (2012). The effect of aging on the memory enhancement of the survival judgment task. Japanese Psychological Research, 54, 210-217. doi:10.1111/j.1468-5884.2011.00483.x

Nouchi, R. (2013). Can the memory enhancement of the survival judgment task be explained by the elaboration hypothesis? Evidence from a memory load paradigm. Japanese Psychological Research, 55, 58-71. doi:10.1111/j.1468-5884.2012.00531.x

Otgaar, H., Jelicec, M., \& Smeets, T. (2015). Identifying the proximate roots of the survival processing advantage. Journal of Psychology, 149, 339-355. doi:10.1080/00223980.2013.879848

Otgaar, H., Peters, M., \& Howe, M. L. (2012). Dividing attention lowers children's but increases adults' false memories. Journal of Experimental Psychology: Learning, Memory, and Cognition, 38, 204-210. doi:10.1037/a0025160

Otgaar, H., \& Smeets, T. (2010). Adaptive memory: Survival processing increases both true and false memory in adults and children. Journal of Experimental Psychology: Learning, Memory, and Cognition, 36, 1010-1016. doi:10.1037/a0019402

Otgaar, H., Smeets, T., \& van Bergen, S. (2010). Picturing survival memories: Enhanced memory after fitness-relevant processing occurs for verbal and visual stimuli. Memory \& Cognition, 38, 23-28. doi:10.3758/MC.38.1.23

Renkewitz, F., \& Müller, S. M. (2015, July 13). Replication of Nairne, Pandeirada, \& Thompson (2008, PS, Study 2). Retrieved from osf.io/jhkpe

Röer, J. P., Bell, R., \& Buchner, A. (2013). Is the survival processing memory advantage due to richness of encoding? Journal of Experimental Psychology: Learning, Memory \& Cognition, 39, 1294-1302. doi:10.1037/a0031214

Seamon, J. G., Goodkind, M. S., Dumey, A. D., Dick, E., Aufseer, M. S., Strickland, S. E., \& Fung, N. S. (2003). "If I didn't write it, why would I remember it?" Effects of encoding, attention, and practice on accurate and false memory. Memory \& Cognition, 31, 445-457. doi:10.3758/BF03194402

Snodgrass, J. G. \& Corwin, J. (1988). Pragmatics of measuring recognition memory: applications to dementia and amnesia. Journal of Experimental Psychology: General, 117, 34-50

Stillman, C. M., Coane, J. H., Profaci, C. P., Howard, J. H., \& Howard, D. V. (2014). The effects of healthy aging on the mnemonic benefit of survival processing. Memory \& Cognition, 42, 175-185. doi:10.3758/s13421-013-0353-2

Suddendorf, T., \& Corballis, M. C. (1997). Mental time travel and the evolution of the human mind. Genetic, Social, and General Psychology Monographs, 123, 133-167.

Tooby, J., \& Cosmides, L. (1992). The psychological foundations of culture. In J. Barkow, L. Cosmides, \& J. Tooby (Eds.), The adapted 
mind: Evolutionary psychology and the generation of culture. New York: Oxford University Press.

Tulving, E. (2002). Episodic memory: From mind to brain. Annual Review of Psychology, 53, 1-25. doi:10.1146/annurev. psych.53.100901.135114
Yang, L., Lau, K. P. L., \& Truong, L. (2014). The survival effect in memory: Does it hold into old age and non-ancestral scenarios? PLoS ONE, 9(5), e95792. doi:10.1371/journal. pone.0095792 